$\mathrm{DTP} / 96 / 10$

February 1996

\title{
Diffractive Heavy Flavour Production at the Tevatron and the LHC
}

\author{
M. Heyssler \\ Department of Physics, University of Durham \\ Durham DH1 3LE, England
}

\begin{abstract}
We give predictions for diffractive heavy flavour production at the Tevatron and the LHC in leading-order approximation. In the framework of these studies we use three different models for the partonic structure of the Pomeron recently proposed by Stirling and Kunszt. These Pomeron models are, despite being fitted to the same diffractive deep inelastic HERA data, very different in their parton content and taken together provide a powerful tool to probe the structure of the Pomeron. All models satisfy GLAP evolution and show a significant $Q^{2}$ dependence. We give numerical predictions for single as well as double diffractive cross sections assuming a Donnachie-Landshoff-type Pomeron flux factor.
\end{abstract}




\section{Introduction}

Recent observations at HERA [1, 2] have provoked a renewed interest in an already long standing concept, the concept of the Pomeron in high energy scattering. This idea was first introduced by Pomeranchuk in 1958 [3] to explain the fact that in the high energy limit the measured hadronic cross sections remain (approximately) constant. This asymptotic behaviour was explained in terms of a Regge trajectory $\alpha(|t|)$ with the Pomeron trajectory having the intercept $\alpha(0) \approx 1$.

Present and future generations of hadron-hadron and lepton-hadron colliders may be able to actually observe the Pomeron in diffractive events. We shall characterise an event as single diffractive if one of the colliding hadrons emits a Pomeron $\mathbb{P}$ that scatters off the other hadron. A single diffractive reaction would therefore correspond to

$$
\text { hadron } i+\text { hadron } j \longrightarrow \text { hadron } i+X \text {, }
$$

in which the intact hadron $i$ emits a Pomeron (hadron $i \rightarrow$ hadron $i+\mathbb{P}$ ) which interacts with hadron $j$ (hadron $j+\mathbb{P} \rightarrow X$ ) leading to the reaction of Eq. (1). Hadron $i$ is detected in the final state with a large longitudinal momentum fraction. Hard diffractive events, events with a large momentum transfer $Q(1 / Q \ll 1 \mathrm{fm})$, are also characterised by a second feature: the absence of hadronic energy in certain angular regions of the final state phase space. These rapidity gaps, reported by the ZEUS and H1 collaborations at HERA, are suggestive of a colour neutral object, the Pomeron, emitted from the incoming proton. Events fulfilling the conditions of large rapidity gaps and a (highly excited) hadron remnant in the final state are called single diffractive to distinguish them from those in which both colliding hadrons remain intact as they each emit a Pomeron, the so-called double diffractive events. The $\mathrm{H} 1$ collaboration reported a fraction of about $6 \%$ of deep inelastic scattering events being single diffractive $\mathbb{4}$. The HERA data suggest that $\mathbb{P}$ has a hard structure. This observation has also been given support in an earlier UA8 experiment. A partonic structure of the Pomeron was first proposed by Ingelman and Schlein in 1985 [5] to calculate high $p_{T}$-jet production in single diffractive dissociation. The discovery of diffractive dijets (high $p_{T}$-jets) with $p_{T}>8 \mathrm{GeV}$ at the CERN $p \bar{p}$-collider in 1988 by the UA8 collaboration [6] fitted exactly with their prediction.

In this paper we give predictions for single and double diffractive heavy flavour production at the Tevatron $p \bar{p}$-collider $(\sqrt{s}=1.8 \mathrm{TeV})$ and the LHC $p p$-collider, with a centre-of-mass energy $\sqrt{s}=10-14 \mathrm{TeV}$. By heavy flavour we mean $Q \bar{Q}$ production with $Q=c$ (charm), $b$ (bottom) or $t$ (top). Even though several tests of diffractive $W$, $Z$ or Higgs production [7, 8, 9, 10] have already been proposed, we shall focus especially on $Q \bar{Q}$ states as their cross sections turn out to be typically larger and this makes their observation, especially in the diffractive context, easier in principle. We shall employ different aspects all deduced from collider physics phenomenology to create a solid model for diffractive scattering via Pomeron exchange. 
The emission of a Pomeron from a fast-moving hadron will be treated in the framework of the Donnachie-Landshoff model [11] of a reggeised, non-perturbative Pomeron. In the literature this type of Pomeron is called soft to discern it from the perturbative (hard) Pomeron. The number of Pomerons emitted by a hadron is described by the flux factor $f_{\mathbb{P} / i}\left(x^{\mathbb{P}},|t|\right)$ where $i$ stands for any hadron. The longitudinal momentum fraction $x^{\mathbb{P}}$ of the Pomeron and the $t$-channel invariant momentum transfer to the Pomeron fully determine the emission factor $f_{\mathbb{P} / i}\left(x^{\mathbb{P}},|t|\right)$. As will be discussed in Section 2, a Regge factor is imbedded in the flux factor instead of a Pomeron propagator.

The success of this prediction has been exploited to expand the ideas of the parton model to the Pomeron. The Pomeron, being a colour neutral object carrying the quantum numbers of the vacuum, should be made of $q \bar{q}$ pairs and/or gluons. Factorisation [12] defines the diffractive structure function $F_{2}^{D}\left(x, Q^{2}, x^{\mathbb{P}},|t|\right)$ as a product of the structure function of the Pomeron $F_{2}^{\mathbb{P}}\left(x, Q^{2}\right)$ and the flux factor, i.e.

$$
F_{2}^{D}\left(x, Q^{2}, x^{\mathbb{P}},|t|\right)=F_{2}^{\mathbb{P}}\left(x, Q^{2}\right) \otimes f_{\mathbb{P} / i}\left(x^{\mathbb{P}},|t|\right) .
$$

Measuring the diffractive structure function $F_{2}^{D}$ and assuming a particular flux (emission) factor $f_{\mathbb{P} / i}\left(x^{\mathbb{P}},|t|\right)$ gives information on the Pomeron structure function $F_{2}^{\mathbb{P}}\left(x, Q^{2}\right)$ and hence on the parton content of the Pomeron. Because factorisation is assumed to give a universal picture of the Pomeron structure, the HERA data on $F_{2}^{\mathbb{P}}\left(x, Q^{2}\right)$ can be used to give predictions for hard diffractive scattering at hadron-hadron colliders. Comparing these predictions with measurements allows the factorisation hypothesis to be tested.

This leads back to the fact that $F_{2}^{D}$ has recently been measured at HERA [13] and thus provides new information on the partonic structure of the Pomeron. The data still suffer from large errors but nevertheless allow a fit of possible parton distributions inside the Pomeron. This has been done for example in Refs. [8, 14, [15]. In particular, in Ref. [8] three Pomeron models were proposed to fit recent H1 data [13]. At this stage it has not been possible to decide experimentally whether the Pomeron is more gluonic or quark-like - deep inelastic scattering (DIS) only gives information on the quark content. Taking this deficiency into account, the three models range from pure glue up to pure quark content at the starting scale $Q_{0}=2 \mathrm{GeV}$. While the quark content in each of the three models turns out to be rather similar, the gluon contents differ from soft to hard. No momentum sum rule for the distributions is imposed and an overall normalisation factor is incorporated into the flux factor, as described in Ref. [15]. A more detailed discussion can be found in Section 3.

The aims of this paper are as follows. We want to show how the combination of Regge theory and factorisation can be applied to calculate diffractive cross sections, especially in the light of recent experimental $F_{2}^{D}$ data. The theory of this is reviewed in Section 2. So far the theory has been mostly applied to single diffractive events. The reason is that

\footnotetext{
${ }^{1}$ The flux factor can also be extracted from experiment.
} 
double diffractive cross sections at present and future colliders are generally too small to play a pivotal or even measurable role. But especially after experiences with top quark production and recent measurements of charm and bottom quark total cross sections at the Tevatron [16] and elsewhere, it is worth looking for heavy flavour production in both single as well as double diffractive cross sections. Future measurements at high energy hadron-hadron colliders together with our predictions could be a powerful step towards a better understanding of the Pomeron structure, in particular to reveal a gluonic, quark-like or mixed Pomeron. We shall also consider the next-generation collider, the LHC, for this purpose. With a centre-of-mass energy $\sqrt{s}=10 \mathrm{TeV}$, we shall show that double diffraction will be no longer an unmeasurable effect but a paradigm for visualising Pomeron-Pomeron scattering.

We note that the concept of a universal factorisation of Pomeron structure, in particular for hadron-hadron collisions, has recently been questioned, see for example Ref. [17]. Our predictions and further experiments at the Tevatron and the LHC might help to clarify this question.

All numerical predictions for the Tevatron and the LHC are presented in Section 4. The results are critically discussed and the different models are carefully discerned. Both absolute values as well as single and double diffractive ratios are shown. Section 5 summarises the results, underlines the most important features and discusses open problems.

\section{Kinematics and Cross Sections}

In this paper we concentrate on the single $\sigma^{S D}$ and double diffractive $\sigma^{D D}$ cross sections for the production of heavy flavour $Q \bar{Q}$ states. What do we mean by diffractive? First let us consider the case of single diffraction, where a Pomeron with momentum $k_{i}^{\mathbb{P}}$ is emitted by one of the colliding hadrons as shown in Fig. 1a. The definition of single diffractive means: one hadron, emitting the Pomeron, is being detected (at least in principle) in the final state. The other hadron scatters off the emitted Pomeron. A typical single diffractive reaction for our purpose at the LHC would read: $p+p \longrightarrow p+Q \bar{Q}+X$. The Pomeron kinematical variable $x_{i}^{\mathbb{P}}$ is defined as: $x_{i}^{\mathbb{P}}=s_{j}^{\mathbb{P}} / s_{i j}$, where $\sqrt{s_{j}^{\mathbb{P}}}$ is the centre-ofmass energy in the Pomeron-hadron $j$ system and $\sqrt{s_{i j}}=\sqrt{s}$ the centre-of-mass energy in the hadron $i$-hadron $j$ system. Instead of the gluon- or (anti)quark-distributions of hadron $i\left(f_{g, q / i}\left(x_{i}, Q^{2}\right)\right)$, the parton distributions of the Pomeron $\left(f_{g, q / \mathbb{P}}\left(x_{i} / x_{i}^{\mathbb{P}}, Q^{2}\right)\right.$ with $x_{i} \leq x_{i}^{\mathbb{P}}$ ) emitted by hadron $i$ define the single diffractive heavy quark cross sections assuming a partonic picture of the Pomeron. We have therefore to substitute

$$
f_{g, q / i}\left(x_{i}, Q^{2}\right) \Rightarrow \int_{\left|t_{i}\right|} d\left|t_{i}\right| \int_{x_{i}^{\mathbb{P}}} \frac{d x_{i}^{\mathbb{P}}}{x_{i}^{\mathbb{P}}} f_{g, q / \mathbb{P}}\left(x_{i} / x_{i}^{\mathbb{P}}, Q^{2}\right) \otimes f_{\mathbb{P} / i}\left(x_{i}^{\mathbb{P}},\left|t_{i}\right|\right) .
$$


The Pomeron parton distribution $f_{g, q / \mathbb{P}}\left(x_{i}^{\mathbb{P}}, Q^{2}\right)$ is weighted by a flux factor $f_{\mathbb{P} / i}\left(x_{i}^{\mathbb{P}},\left|t_{i}\right|\right)$ which gives a measure of the number of Pomerons emitted by hadron $i$. It is therefore a function of the energy loss of the proton $t_{i}=\left(P_{i}-P_{i}^{\prime}\right)^{2}$ due to Pomeron emission as well as of the momentum fraction $x_{i}^{\mathbb{P}}$ carried by the Pomeron. Its form can be modelled by 11

$$
f_{\mathbb{P} / i}\left(x_{i}^{\mathbb{P}},\left|t_{i}\right|\right)=\frac{9 \beta^{2}}{4 \pi^{2}}\left[F_{1}\left(\left|t_{i}\right|\right)\right]^{2}\left(x_{i}^{\mathbb{P}}\right)^{1-2 \alpha\left(\left|t_{i}\right|\right)} .
$$

The coupling of the Pomeron to the proton is assumed to be $3 \beta F_{1}\left(\left|t_{i}\right|\right)$ with $\beta=1.8 \mathrm{GeV}^{-2}$. The factor 3 corresponds to the number of valence quarks inside the hadron. This is discussed and supported in [11]. In fact it should couple in a pointlike form to single quarks. From this point of view the effective Pomeron structure function should contain quarkantiquark contributions. An analogy with the photon structure function emerges from this picture. The elastic form factor of the proton $F_{1}\left(\left|t_{i}\right|\right)$ is experimentally determined and well parametrised by [11]

$$
F_{1}\left(\left|t_{i}\right|\right)=\frac{4 M_{p}^{2}+2.8\left|t_{i}\right|}{4 M_{p}^{2}+\left|t_{i}\right|}\left(1+\frac{\left|t_{i}\right|}{0.7 \mathrm{GeV}^{2}}\right)^{-2},
$$

where $M_{p}$ is the proton mass. Instead of propagators for the Pomeron, one inserts the Regge factor $\left(x_{i}^{\mathbb{P}}\right)^{1-2 \alpha\left(\left|t_{i}\right|\right)}$ with the Regge trajectory $\alpha\left(\left|t_{i}\right|\right)=1+\epsilon-\alpha^{\prime}\left|t_{i}\right|$ with $\epsilon$ and $\alpha^{\prime}$ both deduced experimentally. Latest experiments at the Tevatron 18 showed a slight $\sqrt{s}$ dependence of $\epsilon$ as long as $\alpha^{\prime}$ is fixed. The CDF collaboration studied single diffractive events $p+\bar{p} \rightarrow \bar{p}+X$ and obtained from a data fit:

$$
\begin{aligned}
& \epsilon=0.121 \pm 0.011 \text { at } \quad \sqrt{s}=546 \mathrm{GeV} \\
& \epsilon=0.103 \pm 0.017 \text { at } \sqrt{s}=1800 \mathrm{GeV}
\end{aligned}
$$

They conclude that large screening effects have to be introduced to save the traditional Pomeron model. An analysis at $\sqrt{s}=20,546$ and $1800 \mathrm{GeV}$ showed a significant $\sigma^{S D} \sim$ $s^{0.030}$ dependence for the single diffractive cross section.

In practice, $\epsilon$, introduced as an effective power, measures the deviation of the differential cross section $d \sigma^{S D} / d M^{2}$ from a pure $1 / M^{2}$ dependence. On the other hand, taking only the triple-Pomeron diagram as contribution to diffractive dissociation into account and neglecting screening effects, $1+\epsilon$ can be interpreted as the intercept of a supercritical Pomeron [19].

Also deduced experimentally by CDF was the sensitivity to $\alpha^{\prime}$. A change in $\alpha^{\prime}$ by $\delta \alpha^{\prime}= \pm 0.1 \mathrm{GeV}^{-2}$ results in a change in $\sigma^{S D}$ of only $\pm 0.1 \%$ and $\epsilon$ changes at the same time by $\delta \epsilon= \pm 0.011$. Neglecting any $s$ dependence, we can fix $\epsilon$ and $\alpha^{\prime}$ to the standard values [1] $\epsilon=0.085$ and $\alpha^{\prime}=0.25 \mathrm{GeV}^{-2}$.

According to [11 all these relations should only be valid if we limit the Pomeron momentum fraction to $x_{i}^{\mathbb{P}} \leq 0.1$. Thus only a maximum energy loss of $10 \%$ for each 
hadron is allowed. But assuming the final state proton is not detected, there will be no energy cut for $t$, although the flux factor decreases very fast with $|t|$.

The flux factor plays a pivotal role because it directly yields the energy dependent emission rate of the Pomerons and is therefore extensively discussed in the literature. Bruni and Ingelman calculated the diffractive $W$ production rates at the Tevatron using a constant Pomeron-proton cross section of $2.3 \mathrm{mb}$, obtained from Regge analysis but with a non-Regge parametrisation for the flux factor [9]

$$
f_{\mathbb{P} / i}\left(x_{i}^{\mathbb{P}},\left|t_{i}\right|\right)=\frac{1}{x_{i}^{\mathbb{P}}}\left(6.38 e^{-8\left|t_{i}\right|}+0.424 e^{-3\left|t_{i}\right|}\right) \frac{1}{2.3},
$$

fitted from single diffractive data. Throughout this work, however, we shall use the Donnachie-Landshoff Pomeron flux factorl of Eq. (41).

We shall also consider double diffractive events. The procedure is analogous to the single diffractive scenario and is sketched in Fig. 1b. Now both hadrons undergo Pomeron emission and therefore all hadronic parton distributions are replaced by the parton distributions of the Pomeron via the rule given in Eq. (3). However, the basic formulation of diffractive scattering was intended for reactions where one hadron scatters diffractively and one hadron is highly excited. The crucial point is that the single diffractive cross section of a hadron-hadron collision is assumed to factorise into the total Pomeron-hadron cross section and the Pomeron flux factor [5]. The single diffractive event may then be written as

$$
\frac{d \sigma^{S D}(p+p(\bar{p}) \rightarrow p(\bar{p})+Q \bar{Q}+X)}{d x_{i}^{\mathbb{P}} d\left|t_{i}\right|}=f_{\mathbb{P} / i}\left(x_{i}^{\mathbb{P}},\left|t_{i}\right|\right) \otimes \sigma^{T}(\mathbb{P}+p(\bar{p}) \rightarrow Q \bar{Q}+X) .
$$

Eq. (7) is the analogue of Eq. (3). Factorisation has also been applied to double diffraction, for example predictions for Higgs [10] or $W$ boson production [8, 9] at the Tevatron and/or the LHC. Both colliding hadrons can in principle be detected in the final state. Again, a typical reaction at the LHC would look like: $p+p \longrightarrow p+p+Q \bar{Q}+X$. Double diffractive events thus are characterised by two quasi-elastic protons with rapidity gaps between them and the central heavy flavour products.

Using the substitution given in Eq. (3) we can proceed in complete analogy to the single diffractive case and finally write down the expressions for the total, single and double diffractive cross sections for $Q \bar{Q}$ production

$$
\sigma^{T}\left(s, m_{Q}\right)=\int_{x_{1}=\tau}^{1} \int_{x_{2}=\frac{\tau}{x_{1}}}^{1} d x_{1} d x_{2}\left[f_{q / 1}\left(x_{1}, Q^{2}\right) f_{q / 2}\left(x_{2}, Q^{2}\right) \times \hat{\sigma}_{q \bar{q}}\left(\hat{s}, m_{Q}, \alpha_{S}\left(Q^{2}\right)\right)\right.
$$

\footnotetext{
${ }^{2}$ Goulianos has recently argued 20 that only a normalisation of the flux factor to one Pomeron per nucleon might yield physical results.
} 


$$
\begin{aligned}
& \left.+f_{g / 1}\left(x_{1}, Q^{2}\right) f_{g / 2}\left(x_{2}, Q^{2}\right) \times \hat{\sigma}_{g g}\left(\hat{s}, m_{Q}, \alpha_{S}\left(Q^{2}\right)\right)\right] \\
\sigma^{S D}\left(s, m_{Q}\right) & =\int d x_{1} \int d x_{2} \int \frac{d x_{1}^{\mathbb{P}}}{x_{1}^{\mathbb{P}}} \int d\left|t_{1}\right| \otimes f_{\mathbb{P} / 1}\left(x_{1}^{\mathbb{P}},\left|t_{1}\right|\right) \\
& \times\left[f_{q / \mathbb{P}}\left(x_{1} / x_{1}^{\mathbb{P}}, Q^{2}\right) f_{q / 2}\left(x_{2}, Q^{2}\right) \times \hat{\sigma}_{q \bar{q}}\left(\hat{s}, m_{Q}, \alpha_{S}\left(Q^{2}\right)\right)\right. \\
& \left.+f_{g / \mathbb{P}}\left(x_{1} / x_{1}^{\mathbb{P}}, Q^{2}\right) f_{g / 2}\left(x_{2}, Q^{2}\right) \times \hat{\sigma}_{g g}\left(\hat{s}, m_{Q}, \alpha_{S}\left(Q^{2}\right)\right)\right] \\
& +(1 \rightleftharpoons 2), \\
\sigma^{D D}\left(s, m_{Q}\right) & =\int d x_{1} \int d x_{2} \int \frac{d x_{1}^{\mathbb{P}}}{x_{1}^{\mathbb{P}}} \int \frac{d x_{2}^{\mathbb{P}}}{x_{2}^{\mathbb{P}}} \int d\left|t_{1}\right| \int d\left|t_{2}\right| \otimes f_{\mathbb{P} / 1}\left(x_{1}^{\mathbb{P}},\left|t_{1}\right|\right) f_{\mathbb{P} / 2}\left(x_{2}^{\mathbb{P}},\left|t_{2}\right|\right) \\
& \times\left[f_{q / \mathbb{P}}\left(x_{1} / x_{1}^{\mathbb{P}}, Q^{2}\right) f_{q / \mathbb{P}}\left(x_{2} / x_{2}^{\mathbb{P}}, Q^{2}\right) \times \hat{\sigma}_{q \bar{q}}\left(\hat{s}, m_{Q}, \alpha_{S}\left(Q^{2}\right)\right)\right. \\
& \left.+f_{g / \mathbb{P}}\left(x_{1} / x_{1}^{\mathbb{P}}, Q^{2}\right) f_{g / \mathbb{P}}\left(x_{2} / x_{2}^{\mathbb{P}}, Q^{2}\right) \times \hat{\sigma}_{g g}\left(\hat{s}, m_{Q}, \alpha_{S}\left(Q^{2}\right)\right)\right] .
\end{aligned}
$$

Of course the threshold condition $\hat{s}=x_{1} x_{2} s \geq \tau s=4 m_{Q}^{2}$ for the production of a $Q \bar{Q}$ pair of mass $2 m_{Q}$ must be fulfilled and the cut-off in the Pomeron spectrum $x_{i} \leq x_{i}^{\mathbb{P}} \leq 0.1$ taken into account. With $\hat{s}$ we define the Mandelstam variable of the two contributing subprocesses $q \bar{q} \rightarrow Q \bar{Q}$ and $g g \rightarrow Q \bar{Q}$ and $\tau=4 m_{Q}^{2} / s$ regulates the threshold condition automatically as function of the integration boundaries 3 . We restrict ourselves to the leading-order contributions. The Feynman diagrams of this Born approximation are shown in Fig. 2. A discussion of next-to-leading-order non-diffractive heavy flavour production can be found in [21].

We only cite the expressions for the subprocess cross sections in leading-order approximation. With $\rho=4 m_{Q}^{2} / \hat{s}$ they read

$$
\begin{aligned}
\hat{\sigma}_{g g}\left(\hat{s}, m_{Q}, \alpha_{S}\left(Q^{2}\right)\right) & =\frac{1}{48} \frac{\pi \alpha^{2}\left(Q^{2}\right)}{\hat{s}}(-(112+124 \rho) \sqrt{1-\rho} \\
& \left.+\left(16+16 \rho+\rho^{2}\right) \ln \left(\frac{1+\sqrt{1-\rho}}{1-\sqrt{1-\rho}}\right)\right), \\
\hat{\sigma}_{q \bar{q}}\left(\hat{s}, m_{Q}, \alpha_{S}\left(Q^{2}\right)\right) & =\frac{16}{27} \frac{\pi \alpha^{2}\left(Q^{2}\right)}{\hat{s}}(1+\rho) \sqrt{1-\rho},
\end{aligned}
$$

where the renormalisation scale is taken to be the subprocess collision energy, $Q^{2}=\hat{s}=$ $x_{1} x_{2} s$. For an analytic derivation of Eqs. (11) and (12) using standard techniques we refer the reader to [22, 23, 24].

One might however argue that for the $g g \rightarrow Q \bar{Q}$ subprocess the $\hat{t}$ - and $\hat{u}$-channel contributions should dominate [23], in which case a choice of $Q^{2}=\frac{1}{2}\left(\left(m_{Q}^{2}-\hat{t}\right)+\left(m_{Q}^{2}-\hat{u}\right)\right)$ $=\frac{1}{2} \hat{s}$ might be more reasonable. In fact the parton distributions are affected by the choice of $Q^{2}$ in the framework of GLAP evolution. But as in [24] we could not find a

\footnotetext{
${ }^{3}$ For the sake of simplicity we did not explicitly write down the integration boundaries for $\sigma^{S D}$ and $\sigma^{D D}$. They can easily be deduced from the two conditions: (1) $x_{1} x_{2} \geq \tau$ and (2) $x_{i} \leq x_{i}^{\mathbb{P}} \leq 0.1$.
} 
significant sensitivity of our results to this choice compared to a general $Q^{2}=\hat{s}$ for both subprocesses.

A still unsolved problem is the structure of the Pomeron. Nearly all possible scenarios of parton distributions proposed have favoured a more or less gluonic content. In the following section we shall give a very cursory presentation of the most popular Pomeron models at this stage. A pivotal role will be played by three Pomeron models, recently presented and applied to diffractive $W$ production at the Tevatron and the LHC by Kunszt and Stirling [8]. They will be the input distributions for the diffractive heavy flavour production, which we shall deal with in Section 4.

\section{Models of the Pomeron}

The Pomeron has been postulated to describe hard diffractive collider phenomenology. In the framework of Regge theory a colourless object, carrying the quantum numbers of the vacuum, is able to explain the new observations. A pure gluonic composite seemed to be the simplest explanation on the basis of partonic contents and proposed features [25]. The Pomeron was therefore assumed to behave essentially as a hadron and Ingelman and Schlein introduced the concept of a Pomeron structure function [5].

Further collider experiments, by for example the UA8 collaboration at CERN [6], gave evidence for a hard parton distribution inside the Pomeron but still could not distinguish between a gluon or quark dominated Pomeron.

Another striking problem, not yet fully resolved, was the character of the Pomeron: should it be treated by perturbative or non-perturbative means? Landshoff [26] argued that experimental results are best described by a Pomeron having propagators that are non-perturbative. They must not have a pole at $k^{2}=0$. He summarises the phenomenological features of the so-called soft Pomeron as follows:

- it is rather like a $\mathcal{C}=+1$ isoscalar photon,

- it couples to single quarks with a strength $\beta=1.8 \mathrm{GeV}^{-1}$,

- it is a simple Regge pole with a Regge trajectory $\alpha(|t|)=1+\epsilon-\alpha^{\prime}|t|$ taking into account an effective power $\epsilon$ that decreases with increasing $\sqrt{s}$, but very slowly such that $\epsilon \approx 0.08$ at typical hadron collider energies.

Studies at the $p \bar{p}$-collider at CERN [6] favoured a more hard quark- or gluon-like structure function for the Pomeron than a soft one $\mathrm{A}$. Even though the Pomeron exchange is a pure gluon exchange, this does not imply that the Pomeron structure function is entirely, or even predominantly, gluonic.

\footnotetext{
${ }^{4}$ The conclusion of UA8 was that $\sim 57 \%$ of all events should be hard $[6 x(1-x)]$ and $\sim 13 \%$ were tagged to be soft $\left[6(1-x)^{5}\right]$. The remaining $30 \%$ showed a superhard $[\delta(1-x)]$ characteristic.
} 
This was the starting point to give predictions for diffractive events using different Pomeron models, for example for diffractive $W$ and $Z$ boson production [9] or for diffractive Higgs production [10].

Whatever the parton distributions looked like, in the original investigations they were assumed to show no evolution with the scale $Q^{2}$ at which the Pomeron is probed. They were functions only of the momentum fractions of the partons inside the Pomeron. This situation has changed, since there are now data for the diffractive structure functions measured at HERA [1, 2] at different values of $Q^{2}$. Employing Eq. (2) and assuming factorisation directly yields data for the Pomeron structure function $F_{2}^{\mathbb{P}}\left(x, Q^{2}\right)$, assuming a certain structure for the flux factor. This has been discussed in detail in the Introduction.

We shall focus on the latest set of three different Pomeron models, based on recent HERA data [13], provided by Kunszt and Stirling [8]. The main features of these models are:

- all three models are qualitatively very different concerning the partonic contents at the starting scale $Q_{0}=2 \mathrm{GeV}$,

- all three models give satisfactory agreement with the H1 and ZEUS data,

- all models undergo leading-order GLAP evolution as for the usual parton distributions.

In qualitative terms, the three models can be characterised as:

Model 1: At $Q_{0}$ the Pomeron is entirely composed of quarks. Gluons are dynamically generated via GLAP evolution;

Model 2: A mix of quarks and gluons at starting scale $Q_{0}$;

Model 3: A predominantly hard gluonic content at starting scale, the gluons inside the Pomeron carry large fractional momenta.

An SU(3) flavour symmetry is assumed for all three models, such that a momentum sum rule for $Q_{0}=2 \mathrm{GeV}$ can be imposed

$$
\int_{0}^{1} d x x\left(6 f_{q / \mathbb{P}}\left(x, Q_{0}^{2}\right)+f_{g / \mathbb{P}}\left(x, Q_{0}^{2}\right)\right)=1,
$$

to deduce the overall normalisation factor for the data fit. Because the quark contents inside the three models are more or less comparable since they are constrained by the HERA data, we show in Fig. 3 solely the $Q$ evolution of the gluon distributions $x f_{g / \mathbb{P}}\left(x, Q^{2}\right)$ for the three Pomeron models. 
We shall see later that it is the $g g$ contributions which predominantly govern the behaviour of the (diffractive) $Q \bar{Q}$ cross sections for the Tevatron and the LHC in the given energy regime. These cross sections can therefore in principle provide important information on the gluon content of the Pomeron, complementary to the constraints on the quark content from DIS structure functions. For a broader discussion of the partonic Pomeron contents and their fit to the $\mathrm{H} 1$ data we refer the reader to [8].

It is very important to have distributions that undergo GLAP evolution, because, ranging from charm to top quark masses, different $Q^{2}$ scales are probed and, as can be seen in Fig. 3, the distributions evolve significantly with $Q^{2}$. This is different from most of the former static distributions proposed and enables us to give predictions for such a large mass range covered by the heavy flavours.

The main features of the three Pomeron models concerning $x f_{g / \mathbb{P}}\left(x, Q^{2}\right)$ can easily be deduced from Fig. 3. The left side shows surface plots of the gluon distributions in the regime $4 \mathrm{GeV} \leq Q \leq 50 \mathrm{GeV}$ and $0.1 \leq x \leq 1.0$. The corresponding contour plots in the $x-Q$ plane are presented on the right side.

As can be seen in Fig. 3, Model 1 gains only a small gluon distribution via GLAP evolution at $Q=4 \mathrm{GeV}$. Recall that there are no gluons at all at the starting scale $Q_{0}=2 \mathrm{GeV}$. The gluon distribution is almost flat in $Q$ for moderate and high values of $x$. This fact can also be seen in the contour plot, where for $Q>10 \mathrm{GeV}$ there is only a significant evolution in the very small $x$ regime. No gluons with $x>0.6$ are present in this regime.

This is different to Model 2, which contains gluons with moderate or even higher fractional momenta. The gluon distribution shows a significant $Q$ evolution not only for small but also for moderate $x$ (this can be seen clearly in the contour plot), even though the distribution asymptotically approaches zero for higher $Q$ and $x>0.3$.

Model 3 finally shows the most dynamical evolution of the gluon distributions not only in $Q$ but also in $x$. Very hard gluons are present for high values of $x$. This is due to the basic construction of Model 3 as discussed above. These gluons lose their high momenta at larger values of $Q$ mainly due to quark-antiquark pair production. The depletion of these high fractional-momenta gluons with increasing $Q$ again becomes very transparent in the corresponding contour plot. For a further discussion we again refer the reader to [8].

\section{Diffraction at the Tevatron and the LHC}

For the GLAP evolution of the parton distributions inside the (anti)proton we use the $\operatorname{MRS}\left(\mathrm{A}^{\prime}\right)$ set presented in [27. One test of the reliability of this set is the comparison of the calculated total inclusive cross section for $t \bar{t}$ production at $\sqrt{s}=1.8 \mathrm{TeV}$ with the latest experimental measurement [28]. The CDF collaboration measures a total inclusive cross section of $\sigma_{\text {exp. }}^{T}(p+\bar{p} \rightarrow t \bar{t}+X)=7.6_{-2.0}^{+2.4} \mathrm{pb}$ for an experimentally determined top mass $m_{t}=(176 \pm 8$ (stat.) \pm 10 (syst.) $) \mathrm{GeV}$, while the $\operatorname{MRS}\left(\mathrm{A}^{\prime}\right)$ set of partons predicts 
$\sigma^{T}(p+\bar{p} \rightarrow t \bar{t}+X)=5.81 \mathrm{pb}$ for $m_{t}=176 \mathrm{GeV}$. Measurements of inclusive $c \bar{c}$ and $b \bar{b}$ cross sections at the Tevatron collider yield [16]:

$$
\begin{aligned}
\sigma_{\text {exp. }}^{T}(p+\bar{p} \rightarrow c \bar{c}+X) & =115.2_{-26.8}^{+24.3} \mathrm{nb} \\
\sigma_{\text {exp. }}^{T}(p+\bar{p} \rightarrow b \bar{b}+X) & =30.1_{-17.4}^{+17.0} \mathrm{nb}
\end{aligned}
$$

for $m_{c}=1.5 \mathrm{GeV}$ and $m_{b}=4.5 \mathrm{GeV}$ compared to the leading-order theoretical predictions of $\sigma^{T}(p+\bar{p} \rightarrow c \bar{c}+X)=98.7 \mathrm{nb}$ and $\sigma^{T}(p+\bar{p} \rightarrow b \bar{b}+X)=44.2 \mathrm{nb}$ for the same $m_{c}$ and $m_{b}$. Even though we are working only in leading-order approximation, the agreement with the experimental results is encouraging. Predictions for the ratios $\sigma^{S D} / \sigma^{T}$ and $\sigma^{D D} / \sigma^{T}$, however, should be relatively insensitive to higher-order perturbative corrections.

\subsection{Probing the Pomeron at the Tevatron}

We calculate numerically the total inclusive, the single diffractive and the double diffractive cross sections for heavy flavour pair-production using the $\operatorname{MRS}\left(\mathrm{A}^{\prime}\right)$ set of partons and the three Pomeron models introduced in [B] and discussed in Section 3. Our results are shown in Fig. 4. Supplementary we show the pure gluon fusion contribution as dashed lines.

For the Tevatron collider the $q \bar{q}$ process becomes dominant for $m_{Q}>50 \mathrm{GeV}$. This $q \bar{q}$ dominance is obviously visible in the total cross section but becomes even more striking for the single diffractive one. Due to this process, the single diffractive ratio $R^{S D}$ defined as $R^{S D}=\sigma^{S D} / \sigma^{T}$ even increases for $m_{Q}>70 \mathrm{GeV}$ before this process runs out of centre-of-mass energy $\sqrt{\hat{s}}$ due to the cut-off in the Pomeron spectrum $\left(x_{i}^{\mathbb{P}} \leq 0.1\right)$ and steeply falls to zero. Note that this restriction on $\sqrt{\hat{s}}$ unfortunately takes place before the top quark production domain is reached. The single diffractive ratio $R^{S D}$ for all three Pomeron models reaches its local maximum at slightly lower masses than the top quark mass. This gives little hope to observe diffractive top quark events at the Tevatron but is promising for the LHC as will be discussed in Section 4.2.

At Tevatron energy the threshold for single diffractive events is reached when $m_{Q}=$ $270 \mathrm{GeV}$. But single diffractive events might be observed only up to $m_{Q} \approx 150 \mathrm{GeV}$ with

$$
\begin{aligned}
& \text { Model 3: } \sigma_{\text {max. }}^{S D}=1.05 \mathrm{pb} \simeq 4.05 \% \text { of } \sigma^{T}, \\
& \text { Model 1: } \sigma_{\min .}^{S D}=0.22 \mathrm{pb} \simeq 0.86 \% \text { of } \sigma^{T}
\end{aligned}
$$

for $m_{Q}=150 \mathrm{GeV}$. This is qualitatively what we expect. Model 3 with the hard gluon content gains more and more $q \bar{q}$ pairs via GLAP evolution which dominantly contribute to the single diffractive cross section for higher masses. Model 2 with the quark-gluon mixture dominates for small masses, but at $m_{Q} \simeq 10 \mathrm{GeV}$ Model 3 takes over. The production rate of the important $q \bar{q}$ pairs for higher masses increases more rapidly in Model 3. Model 1, however, without any gluons at the initial $Q_{0}=2 \mathrm{GeV}$ scale, marks 
the lower limit for single and double diffractive scattering over the whole mass range. The numerical values for the single and double diffractive production of charm, bottom and top are given in Table 1.

Let us now focus on Models 2 and 3: the ratios for single diffractive scattering range between $\sim 22 \%$ for charm and $\sim 15 \%$ for bottom quark production and reach their locally lowest values of $\sim 2 \%$ for $m_{Q}=70 \mathrm{GeV}$, before the ratio even increases due to the $q \bar{q}$ contribution in the mass region $70 \mathrm{GeV} \leq m_{Q} \leq 150 \mathrm{GeV}$ locally peaking at $m_{Q}=150 \mathrm{GeV}$ with ratio $R^{S D}=5.5 \%(4.2 \%)$ for Model 3 (2). Qualitatively Model 1 shows the same behaviour. But with only quarks in the starting distribution, the creation of gluons and $q \bar{q}$ pairs via GLAP evolution proceeds only slowly. This can be seen in Fig. 3. The difference is expressed in the large gap between Model 1 on the one hand and Models 2 and 3 on the other hand. So the conclusion is that at least for single diffraction a gluon rich Pomeron as input should be more easily detected. Model 1 and Model 3 for example differ by about one order of magnitude. This turns out to be a crucial difference with such absolutely small diffractive cross sections.

Double diffractive scattering seems to favour a balanced mixture of quarks and gluons in the starting distribution and during GLAP evolution, as provided in Model 2. This can again be observed in Fig. 4.

Notice that the former dominant hard gluon Model 3 quantitatively shows about the same behaviour as Model 1. Why the behaviour of Model 3 in double diffraction is different from that in single diffraction can be understood by a further analysis of the kinematics among the partons inside the Pomeron and their distributions at different mass scales. Models 2 and 3 show a crossing in the case of single diffractive scattering as can be observed in Fig. 4. Its existence can be immediately explained in terms of the gluon distributions which are shown in Fig. 3. As we have already pointed out, the gluon distributions govern the behaviour of the cross sections at the Tevatron, especially for small quark masses. For small $x$ and $Q$, the gluon distribution of Model 2 is slightly bigger than that of Model 3. For higher $x$ and/or $Q$ the situation is reversed.

In the case of double diffraction, an analysis of the average fractional gluon momentum $\langle x\rangle=\left\langle x_{i} / x_{i}^{\mathbb{P}}\right\rangle$ yields $\langle x\rangle \sim 0.11$ for $m_{Q}=2 \mathrm{GeV}$ and $\langle x\rangle \sim 0.24$ for $m_{Q}=50 \mathrm{GeV}$ for all three models. In this regime the gluon distribution of Model 2 again exceeds that of Model 3. So, no crossing can be observed and Model 2 dominates throughout. In fact the crossing would take place at $m_{Q} \sim 80 \mathrm{GeV}$, shortly before the threshold for double diffraction with $m_{Q} \sim 90 \mathrm{GeV}$ is reached.

The relatively broad gap between Model 2 and Model 3 appearing in Fig. 4 can again be explained by the same straightforward analysis of the average gluon momentum. Again a comparison with the corresponding gluon distributions of Model 2 and Model 3 in Fig. 3 shows the absolute difference of these distributions in the regime $0.1 \leq\langle x\rangle \leq 0.2$.

While diffractive charm and bottom quark production might be observable at the Tevatron, there is no hope for diffractive $t \bar{t}$ pair production being visible at a total centre- 
of-mass energy of $\sqrt{s}=1.8 \mathrm{TeV}$. Either the top mass exceeds the kinematic threshold (double diffraction) or the effect of the threshold is already strongly influencing the process by a steep decrease in the diffractive cross section (single diffraction near top mass). However at the LHC such heavy flavour threshold suppression is less severe, as we shall see in the following Section.

\subsection{Predictions for the LHC}

The LHC will provide a rich field of study for diffractive events, even in the top mass regime. With a centre-of-mass energy of at least $\sqrt{s}=10 \mathrm{TeV}$ [29] the double diffractive threshold is reached for $m_{Q}=500 \mathrm{GeV}$ and the single diffractive one lies at $m_{Q}=$ $1500 \mathrm{GeV}$. This upper bound is very promising in particular for diffractive top quark production at the LHC.

At a proton-proton collider, the dominant process for $Q \bar{Q}$ production is of course gluon-gluon fusion since antiquarks only appear as sea quarks inside the proton. For a top mass of $176 \mathrm{GeV}$ the pure $g g$ contribution is about $91 \%$ of the total cross section in our calculation. This also holds for the single and double diffractive case.

The numerical results are shown in Fig. 5 and the numerical values are again listed in Table 1. The single diffractive ratios $R^{S D}$ for the three Pomeron models are between $20-40 \%$ for charm and $10-40 \%$ for bottom quarks. The maximal and minimal single diffractive top quark rates are

$$
\begin{aligned}
& \text { Model 3: } \sigma_{\max }^{S D}=5.45 \mathrm{pb} \simeq 1.66 \% \text { of } \sigma^{T} \text {, } \\
& \text { Model 1: } \sigma_{\text {min. }}^{S D}=1.13 \mathrm{pb} \simeq 0.34 \% \text { of } \sigma^{T}
\end{aligned}
$$

with $m_{t}=176 \mathrm{GeV}$. Even though the single diffractive ratios for top production are comparable to the Tevatron rates, the absolute single diffractive cross sections are crucially enhanced. We find an enhancement of about a factor 100 at the LHC. For example Model 2 and Model 3 provide a single diffractive cross section of approximately $5 \mathrm{pb}$. This is about the total cross section for top production at the Tevatron. So, single diffractive top quark events should be readily detected at the LHC.

The predictions for double diffractive scattering, however, are still not very promising. The maximal double diffractive cross section (for Model 2) is $1.86 \cdot 10^{-2} \mathrm{pb}$ for top quarks. The qualitative behaviour of the three different Pomeron models is the same. Because the centre-of-mass energy at the LHC is larger by a factor of six, one can conclude from the qualitatively similiar behaviour of all three models at the LHC that the gluon distributions in all three models do indeed become similiar at higher $Q$, as already seen in Fig. 3 .

All conclusions that were drawn for the Tevatron still hold for the LHC, except that because of the higher centre-of-mass energy there are no kinematical artifacts in the considered flavour mass regime. Even for the top quark mass, the single as well as the 
double diffractive cross section behave rather smoothly. The influence of the threshold does not seriously affect the cross sections in this case.

Considering the ratios for single and double diffractive events in Fig. 5 again shows a qualitatively comparable picture to the Tevatron. Again an analysis of the average fractional gluon momentum $\langle x\rangle$ will explain the differences in $R^{S D}$ and $R^{D D}$. For $R^{S D}$, Models 2 and 3 are quantitatively equivalent, especially for the charm and bottom quarks. For a bottom quark mass of $m_{b}=4.5 \mathrm{GeV}$, we obtain as average fractional gluon momentum $\langle x\rangle=0.42$ for $Q \sim 2 m_{b}=9 \mathrm{GeV}$ in the single diffractive case. A comparison with the gluon distributions in Fig. 3 shows that they are roughly equal for Model 2 and Model 3 in this region of $x$. This fact becomes even more transparent in the contour plots of Fig. 3.

For the case of double diffraction the same analysis yields a lower average fractional momentum for the gluons, due to the energy-cut among both Pomeron emitting hadrons. Again for $m_{b}=4.5 \mathrm{GeV}$ we obtain $\langle x\rangle=0.22$. But in this region of $x$, the gluon distribution of Model 3 shows a local minimum, the hard gluons in this model give a rise of $x f_{g / \mathbb{P}}\left(x, Q^{2}\right)$ only for $x>0.5$ in the low-Q regime. The gluon distributions inside Model 2 also show a local minimum around $x \sim 0.2$, but its absolute value is higher than that for Model 3 in this region. This fact is responsible for the gap between Model 2 and Model 3 as observed for $R^{D D}$ in Fig. 5. For higher quark masses (higher values of $Q^{2}$ ) all three models become comparable concerning the gluon distributions, as already discussed.

Model 2 and Model 3 as descriptions of the parton distributions of the Pomeron yield very promising single diffractive ratios, at least for charm and bottom quarks. Model 3 predicts a single diffractive ratio of $\sim 40 \%$ for $c \bar{c}$ and $b \bar{b}$ production. This is quantitatively comparable to the predictions of Model 2 as can be observed in Fig. 5 and numerically verified in Table 1. Thus about one third of the production of heavy flavours at the LHC including charm and bottom quarks should be single diffractive. But even Model 1, purely quark-like at starting scale $Q_{0}=2 \mathrm{GeV}$, gives a single diffractive contribution of approximately $20 \%$ in this mass regime.

\section{Conclusions}

We have presented predictions for diffractive heavy flavour production at the Tevatron and the LHC. The input Pomeron distributions [8] were different in contents from pure quark starting distributions up to hard gluonic contents at the GLAP starting scale $Q_{0}=2 \mathrm{GeV}$. Our predictions can therefore be considered as upper and lower limits for the diffractive cross sections.

We found that at the Tevatron single and double diffractive charm and bottom quark production is observable with a single diffractive ratio $R^{S D}$ that is between $7 \%$ (charm) and $4 \%$ (bottom) for Model 1 as a lower limit and 26\% (charm) and 13\% (bottom) for 
Model 2 as an upper bound. The double diffractive cross section for charm and bottom production lies above the total cross section for the top quark and might also be observable. The lower limit is governed by the pure quark-like Model 1 with $\sigma^{D D} \sim 45 \mathrm{pb}$ for charm and $\sigma^{D D} \sim 18 \mathrm{pb}$ for bottom quarks, whereas having Model 2 as the underlying Pomeron model gives a double diffractive cross section which is enhanced by a factor of four.

The diffractive cross sections for charm and bottom quarks will be larger by three orders of magnitude at the LHC, and also single diffractive top quark production should be observable. The single diffractive $t \bar{t}$ cross sections range from $1 \mathrm{pb}$ for Model $1 \mathrm{up}$ to $5 \mathrm{pb}$ for the hard gluon Model 3, which is roughly the total top quark cross section at the Tevatron. All predictions are collected in Table 1 and shown in Figs. 4 and 5.

The three Pomeron models presented in [8] and used in our calculations are only a guide to possible Pomeron structures. Their determinations have been made possible by two strong assumptions: first, the Pomeron has a partonic structure and second, we assume factorisation such that recent measurements of the diffractive Pomeron structure function give a universal picture of the Pomeron. Only the latter makes predictions for the hadron-hadron collider meaningful.

Our predictions can only be a guide as the calculations are illustrative only. We need a more detailed study with detector acceptance. This will follow as soon as there are first results on diffractive heavy flavour production at the Tevatron. But a study of these events $i s$ worthwhile, at least for diffractive charm and bottom production. Diffractive top quark events still will require a higher centre-of-mass energy before they can be studied. The LHC will be such a laboratory for diffractive scattering studies. We predict that about $30 \%$ of all charm and bottom production events should be single diffractive.

It is not yet certain whether the theoretical framework of the Donnachie-Landshoff model can be extended to double or even multiple Pomeron exchange. Again, we give predictions for the case of an analogue treatment of double Pomeron exchange that will be interesting to test at future hadron-hadron colliders. Also the parameters used in this theoretical model have to be fitted to new high energy collider experiments. Finally future high energy experiments will hopefully more clearly reveal the structure of the Pomeron and answer some questions that were raised in this paper.

\section{Acknowledgements}

I would like to thank James Stirling for suggesting the problem and many fruitful discussions throughout this work. John Campbell is thanked for a critical reading of this manuscript. Financial support in the form of a "DAAD-Doktorandenstipendium" HSPII/AUFE is gratefully acknowledged.

\footnotetext{
${ }^{5}$ Especially we expect the effective power $\epsilon$ inside the Pomeron flux factor to become smaller for typical LHC energies.
} 


\section{References}

[1] H1 Collaboration: T. Ahmed et al., Nucl. Phys. B429 (1994) 477.

[2] ZEUS Collaboration: M. Derrick et al., Phys. Lett. B315 (1993) 481; Phys. Lett. B332 (1994) 228; Phys. Lett. B338 (1994) 483.

[3] I.Y. Pomeranchuk, Sov. Phys.-JETP 7 (1958) 499.

[4] H1 Collaboration: T. Ahmed et al., Phys. Lett. B298 (1992) 469.

[5] G. Ingelman and P. Schlein, Phys. Lett. B152 (1985) 256.

[6] UA8 Collaboration: R. Bonino et al., Phys. Lett. B211 (1988) 239. UA8 Collaboration: A. Brandt et al., Phys. Lett. B297 (1992) 417.

[7] D. Graudenz and G. Veneziano, Phys. Lett. B365 (1996) 302. A. Bialas and P.V. Landshoff, Phys. Lett. B256 (1991) 540.

A. Donnachie and P.V. Landshoff, Nucl. Phys. B303 (1988) 634.

K.J. Foley and P. Schlein, Proceedings of the '1986 Summer Study on the Physics of the SSC', Snowmass, Colorado, 1986.

A.R. White, 'Diffractive Heavy Flavour Production including $W^{ \pm}$and $Z^{0}$ ', talk given at the Workshop on Physics Simulations at High Energy, Madison, Wisconsin, 1986.

[8] Z. Kunszt and W.J. Stirling, 'The Parton Interpretation of Hard Diffractive Scattering', presented at the Workshop on HERA Physics, Durham, 1995.

[9] P. Bruni and G. Ingelman, Phys. Lett. B311 (1993) 317.

[10] O. Nachtmann, A. Schäfer and R. Schöpf, Phys. Lett. B249 (1990) 331.

[11] A. Donnachie and P.V. Landshoff, Nucl. Phys. B244 (1984) 322; Nucl. Phys. B267 (1985) 690.

[12] A. Donnachie and P.V. Landshoff, Phys. Lett. B191 (1987) 309; Phys. Lett. B198 (1987) 590.

G. Ingelman and K. Janson-Prytz, Proceedings of the 'Physics at HERA Workshop', eds. W. Buchmüller and G. Ingelman, Hamburg 1991, p. 233.

G. Ingelman and K. Janson-Prytz, Z. Phys. C58 (1993) 285.

[13] H1 Collaboration: T. Ahmed et al., Phys. Lett. B348 (1995) 681.

ZEUS Collaboration: M. Derrick et al., Z. Phys. C68 (1995) 569. 
[14] H Fritzsch and K.H. Streng, Phys. Lett. B164 (1985) 391.

N. Artega-Romero, P. Kessler and J. Silva, Mod. Phys. Lett. A1 (1986) 211; Mod. Phys. Lett. A4 (1989) 645.

E.L. Berger, J.C. Collins, D.E. Soper and G. Sterman, Nucl. Phys. B286 (1987) 704.

J. Bartels and G. Ingelman, Phys. Lett. B235 (1990) 175.

A. Capella et al., 'Hard Diffraction at HERA and the Gluonic Content of the Pomeron', preprint LPTHE-ORSAY-95-41, 1995.

K. Golec-Biernat and J. Kwieciński, Phys. Lett. B353 (1995) 329.

[15] T. Gehrmann and W.J. Stirling, 'Deep Inelastic Electron-Pomeron Scattering at HERA', preprint DTP/95/26, 1995, to be published in Z. Phys. C.

[16] CDF Collaboration: F. Abe et al., Phys. Rev. Lett. 69 (1992) 3704.

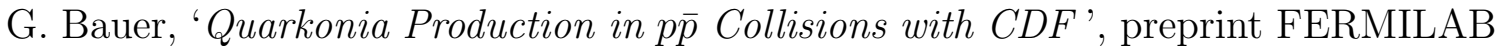
Conf-95/175-E, 1995.

CDF Collaboration: F. Abe et al., ' $\Upsilon$ Production at $C D F$ ', preprint FERMILAB Conf-95/227-E, 1995.

D0 Collaboration: S. Abachi et al., 'J/ $\Psi$ Production in $p \bar{p}$ Collisions at $\sqrt{s}=$ 1.8 TeV', preprint FERMILAB Conf-95/205-E, 1995.

[17] A. Berera and D.E. Soper, 'Behavior of Diffractive Parton Distribution Functions', preprint PSU-TH-163, 1995.

[18] CDF Collaboration: F. Abe et al., Phys. Rev. D50 (1994) 5535.

[19] A.R. White, 'Buchmüller Scaling, the QCD Pomeron, and Tevatron Diffractive Hard Scattering', preprint ANL-HEP-PR-95-57, 1995.

[20] K. Goulianos, 'Pomeron Flux Renormalization in Soft and Hard Diffraction', preprint RU 95/E-06, 1995.

[21] P. Nason, 'Heavy Quark Production', in: 'Heavy Flavours', eds. A.J. Buras and M. Lindner (World Scientific, Singapore 1992) p. 556.

S. Dawson, R.K. Ellis and P. Nason, Nucl. Phys. B303 (1988) 607.

[22] L.M. Jones and H.W. Wyld, Phys. Rev. D17 (1978) 1782.

[23] M. Glück, J.F. Owens and E. Reya, Phys. Rev. D17 (1978) 2324.

[24] B.L. Combridge, Nucl. Phys. B151 (1979) 429.

[25] F.E. Low, Phys. Rev. D12 (1975) 163.

S. Nussinov, Phys. Rev. Lett. 34 (1975) 1286; Phys. Rev. D14 (1976) 246. 
[26] P.V. Landshoff, 'The Structure of the Pomeron', talk given at the XXVII Recontre de Moriond, 1992.

[27] A.D. Martin, W.J. Stirling and R.G. Roberts, Phys. Lett. B354 (1995) 155.

[28] A. Beretvas, 'CDF Results on Top', preprint FERMILAB-PUB-95/310-E, 1995.

[29] Proceedings of the 'Large Hadron Collider Workshop', eds. G. Jarlskog and D. Rein, Aachen 1990. 


\begin{tabular}{|c|c|c|c|c|c|c|}
\hline & \multicolumn{3}{|c|}{ Tevatron $(\sqrt{s}=1.8 \mathrm{TeV})$} & \multicolumn{3}{|c|}{$\mathrm{LHC}(\sqrt{s}=10.0 \mathrm{TeV})$} \\
\hline \multirow[b]{2}{*}{ model } & \multicolumn{3}{|c|}{$\left\langle m_{c}\right\rangle$} & \multicolumn{3}{|c|}{$\left\langle m_{c}\right\rangle$} \\
\hline & 1 & 2 & 3 & 1 & 2 & 3 \\
\hline$\sigma^{S D}[\mathrm{pb}]$ & $7.81 \cdot 10^{3}$ & $29.82 \cdot 10^{3}$ & $24.32 \cdot 10^{3}$ & $2.07 \cdot 10^{6}$ & $3.59 \cdot 10^{6}$ & $3.78 \cdot 10^{6}$ \\
\hline$R^{S D}[\%]$ & 6.83 & 26.15 & 21.27 & 21.43 & 37.21 & 39.21 \\
\hline$\sigma^{D D}[\mathrm{pb}]$ & 45.32 & 208.12 & 60.68 & $4.05 \cdot 10^{4}$ & $6.56 \cdot 10^{4}$ & $4.44 \cdot 10^{4}$ \\
\hline$R^{D D}[\%]$ & $3.96 \cdot 10^{-2}$ & $18.21 \cdot 10^{-2}$ & $5.31 \cdot 10^{-2}$ & 0.42 & 0.68 & 0.46 \\
\hline \multirow[b]{2}{*}{ model } & \multicolumn{3}{|c|}{$\left\langle m_{b}\right\rangle$} & \multicolumn{3}{|c|}{$\left\langle m_{b}\right\rangle$} \\
\hline & 1 & 2 & 3 & 1 & 2 & 3 \\
\hline$\sigma^{S D}[\mathrm{pb}]$ & $3.07 \cdot 10^{3}$ & $11.59 \cdot 10^{3}$ & $10.68 \cdot 10^{3}$ & $4.50 \cdot 10^{5}$ & $1.01 \cdot 10^{6}$ & $1.11 \cdot 10^{6}$ \\
\hline$R^{S D}[\%]$ & 3.31 & 12.49 & 11.51 & 15.50 & 34.53 & 38.27 \\
\hline$\sigma^{D D}[\mathrm{pb}]$ & 17.82 & 78.85 & 25.26 & $5.81 \cdot 10^{3}$ & $1.51 \cdot 10^{4}$ & $7.55 \cdot 10^{3}$ \\
\hline$R^{D D}[\%]$ & $1.92 \cdot 10^{-2}$ & $8.49 \cdot 10^{-2}$ & $2.72 \cdot 10^{-2}$ & 0.20 & 0.52 & 0.26 \\
\hline \multirow[b]{2}{*}{ model } & \multicolumn{3}{|c|}{$\left\langle m_{t}\right\rangle$} & \multicolumn{3}{|c|}{$\left\langle m_{t}\right\rangle$} \\
\hline & 1 & 2 & 3 & 1 & 2 & 3 \\
\hline$\sigma^{S D}[\mathrm{pb}]$ & $1.86 \cdot 10^{-2}$ & $6.06 \cdot 10^{-2}$ & $8.72 \cdot 10^{-2}$ & 1.13 & 4.10 & 5.45 \\
\hline$R^{S D}[\%]$ & 0.31 & 1.01 & 1.45 & 0.34 & 1.25 & 1.66 \\
\hline$\sigma^{D D}[\mathrm{pb}]$ & 0 & 0 & $\bigcirc$ & $1.04 \cdot 10^{-4}$ & $1.86 \cdot 10^{-2}$ & $3.71 \cdot 10^{-3}$ \\
\hline$R^{D D}[\%]$ & 0 & 0 & 0 & $3.17 \cdot 10^{-4}$ & $5.68 \cdot 10^{-3}$ & $1.13 \cdot 10^{-3}$ \\
\hline
\end{tabular}

Table 1: The values for single and double diffractive cross sections, as well as their ratios to the total cross sections, are shown for average quark masses $\left\langle m_{c}\right\rangle=1.3 \mathrm{GeV}$, $\left\langle m_{b}\right\rangle=4.3 \mathrm{GeV}$ and $\left\langle m_{t}\right\rangle=176 \mathrm{GeV}$. The three different Pomeron models are discussed in Section 3. We obtain numerical data for both the Tevatron and the LHC. A $\bigcirc$ indicates that the threshold for this process was exceeded. 


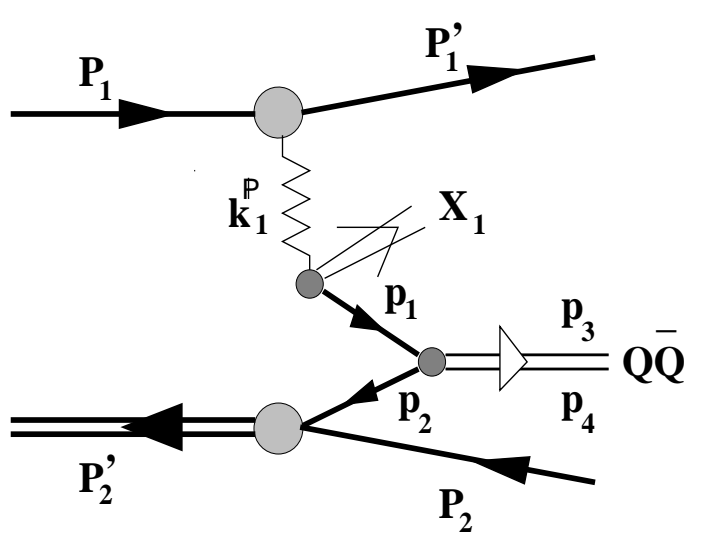

a)

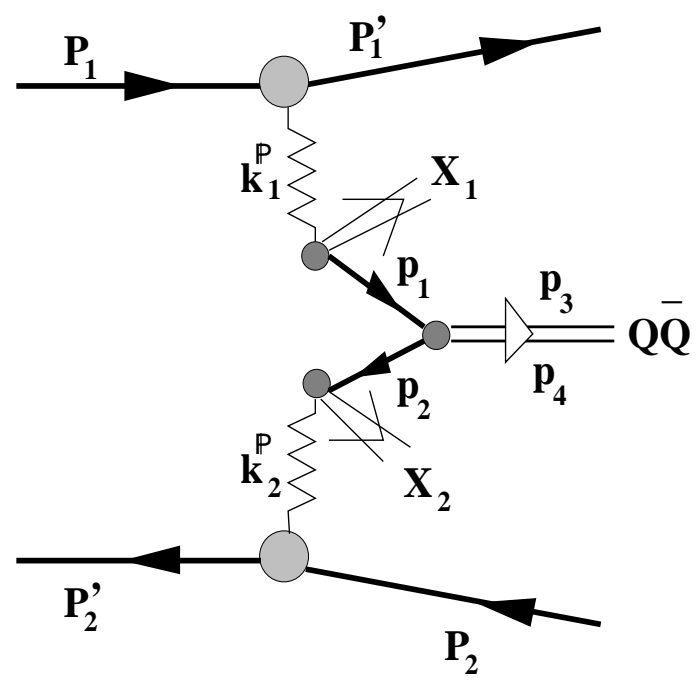

b)

Figure 1: Kinematics of the single (a) and the double diffractive scattering (b) events, leading to $Q \bar{Q}$ pair production. The subprocesses taken into account are either $q \bar{q} \rightarrow Q \bar{Q}$ or $g g \rightarrow Q \bar{Q}$. 


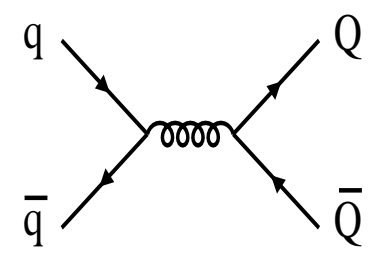

a)
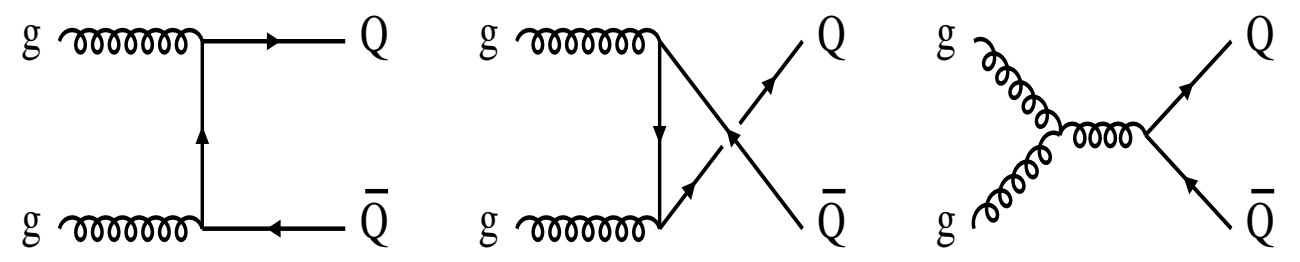

b)

Figure 2: The leading-order Feynman diagrams for the subprocesses $q \bar{q} \rightarrow Q \bar{Q}$ (a) and $g g \rightarrow Q \bar{Q}(\mathbf{b})$. 

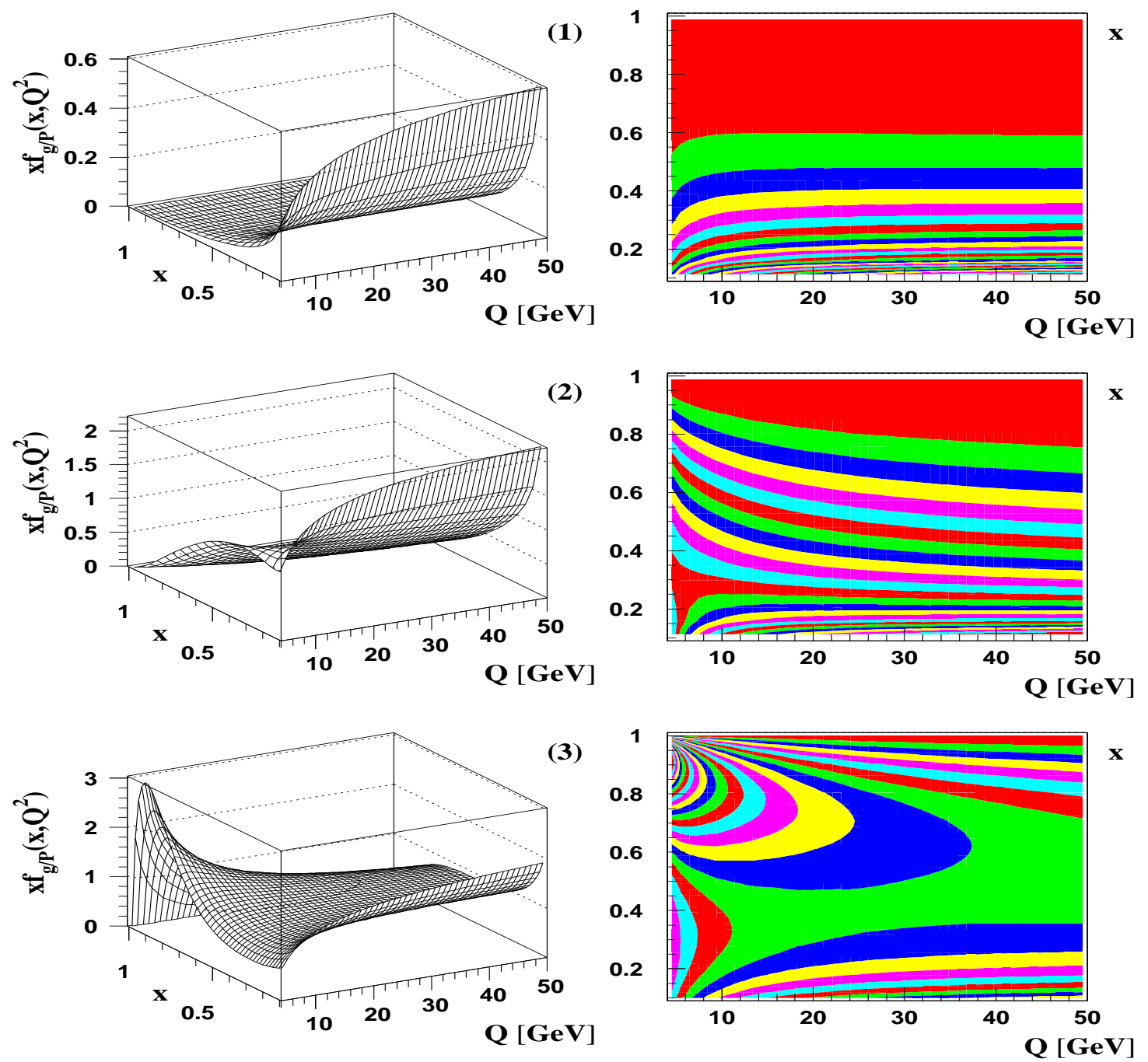

Figure 3: The gluon distributions of the three different Pomeron models are shown, starting with Model 1 at the top. For each model we show the surface plot for a given $x-Q$ regime as well as the corresponding contour plot in the $x-Q$ plane. 

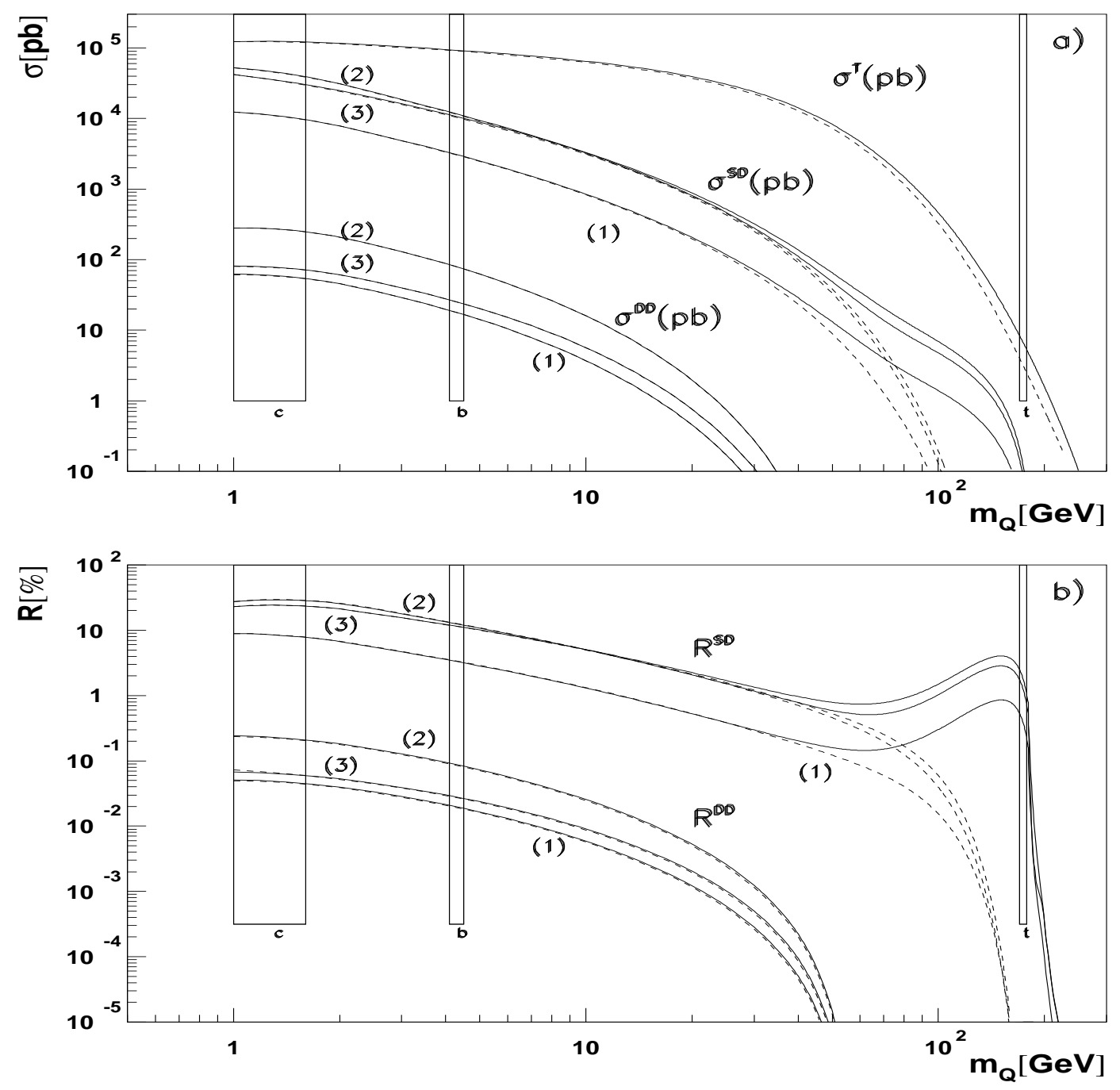

Figure 4: Diffractive Scattering at the Tevatron: The numerical results of the total, single and double diffractive cross sections for the three Pomeron models are shown. The solid lines indicate the subprocess $g g+q \bar{q} \rightarrow Q \bar{Q}$, the dashed lines show solely the contribution from gluon fusion $(g g \rightarrow Q \bar{Q})$ : (a) gives the absolute numbers for the cross sections (in $\mathrm{pb}$ ), and (b) gives the ratios $\left(R^{S D}=\sigma^{S D} / \sigma^{T}\right.$ and $\left.R^{D D}=\sigma^{D D} / \sigma^{T}\right)$. The mass regions of the charm (c), bottom (b) and top (t) quarks are indicated. The fixed centre-of-mass energy is $1.8 \mathrm{TeV}$. 

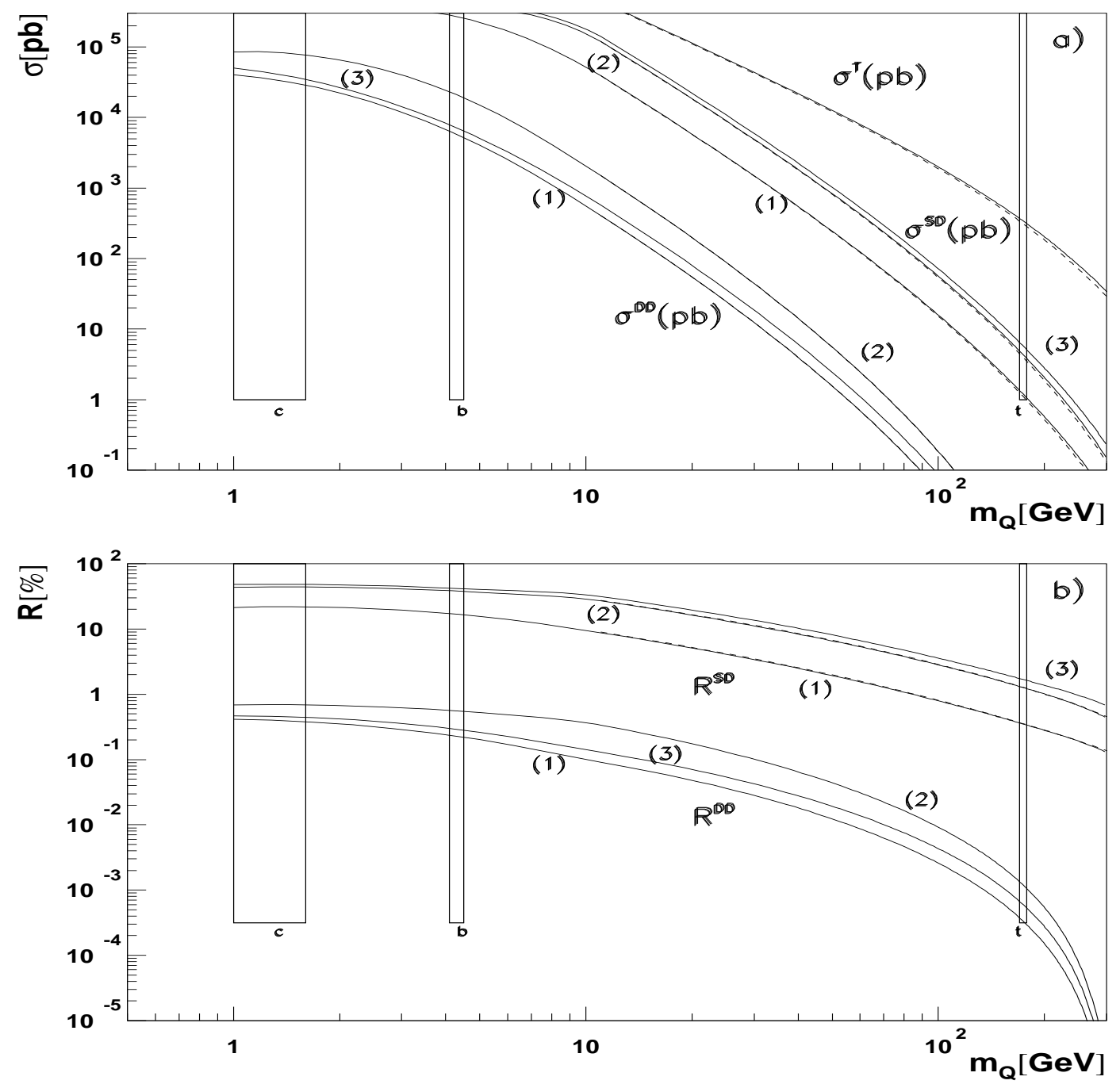

Figure 5: Diffractive Scattering at the LHC: Same as Fig. 4 but now for a centre-of-mass energy of $10 \mathrm{TeV}$. The solid lines indicate the subprocess $g g+q \bar{q} \rightarrow Q \bar{Q}$, the dashed lines show solely the contribution from gluon fusion $(g g \rightarrow Q \bar{Q})$ : (a) gives the absolute numbers for the cross sections (in pb), and (b) gives the ratios $\left(R^{S D}=\sigma^{S D} / \sigma^{T}\right.$ and $\left.R^{D D}=\sigma^{D D} / \sigma^{T}\right)$. 